\title{
EAl Endorsed Transactions

\section{Using Resonance and Soft-Switching to Enhance Efficiency of Underwater Vehicle's Contactless Charge System}

\author{
V.A. Gerasimov ${ }^{1}$, A.V. Komlev² and A.Yu. Filozhenko ${ }^{1}$ \\ ${ }^{1}$ Institute of Marine Technology Problems, Far Eastern Branch of the Russian Academy of Sciences, Russian Federation, \\ imtp@ marine.febras.ru \\ ${ }^{2}$ Far Eastern Federal University, Russian Federation, rectorat@dvfu.ru
}

\section{Abstract}

The contactless battery charge system of the autonomous underwater vehicle allows to significantly increase the device operating time in automatic mode under water. Using a transformer with a non-magnetic gap in such a system leads to an increased load on the inverter's power switches. The article presents a solution to the problem of unloading switches using resonant circuits in combination with the "soft switching" mode of the inverter. It is proposed to use a serial LC-circuit connected in parallel to the transformer primary winding, and a capacitor on its secondary side. Recommendations are given for selecting parameters of resonant elements that minimize the charge time of the underwater vehicle's batteries. The using of research results allows to significantly reduce heat loss while maintaining the transmitted active power. The losses in the inverter were reduced from $130 \mathrm{~W}$ to $12 \mathrm{~W}$ for the transformer idle mode, and from $170 \mathrm{~W}$ to $25 \mathrm{~W}$ for the transformer short-circuit mode.

Keywords: inverter, contactless power transmission, autonomous underwater vehicle, soft-switching, series resonant circuit, parallel resonance.

Received on 09 June 2020, accepted on 30 July 2020, published on 04 August 2020

Copyright (C 2020 V.A. Gerasimov et al., licensed to EAI. This is an open access article distributed under the terms of the Creative Commons Attribution licence (http://creativecommons.org/licenses/by/3.0/), which permits unlimited use, distribution and reproduction in any medium so long as the original work is properly cited.

doi: 10.4108/eai.1-7-2020.165961

*Corresponding author. Email: Kom86@bk.ru

\section{Introduction}

The source of energy for an autonomous underwater vehicle (AUV) in most of its applications is rechargeable batteries, which can be charged in various ways [1]-[4]. Usually, the AUV is lifted on aboard the providing vessel to charge the batteries. It's simplest method for hardware implementation, but technologically the most timeconsuming. Increasing the speed of this process with a significant expansion of the AUV functionality is obtained when charging its batteries using a contactless method of transmitting electricity. This process involves a special transformer with separate primary and secondary parts. The primary part of such a transformer is placed on the base, and the secondary part is installed on the AUV.
A DC-AC converter (inverter) is used to power the primary winding of the transformer. It converts the power of the base source to a high-frequency voltage and also provides the necessary regulation of the transmitted electrical energy [4].

Various underwater and coastal objects can be used as a base. This can be, for example, the so-called "garage", lowered from the supply vessel under water. In this case, the transmission of electricity to the inverter is carried out via a cable. The cable, in addition to electrical communication, provides mechanical power communication between the vessel and the "garage" and the information channel between the operator on the supply vessel and the AUV. Some tasks of using AUVs require the organization of its long-time underwater basing. For example, it may be port security or monitoring 
the underwater situation of a given area. In this case, the design of the mooring base for AUV is performed in the form of bottom dock-station (BDS), installed stationary on the seabed. The power supply of the BDS can be carried out using a cable that connects the BDS to the coastal control centre (CCC). In this case, along with the transmission of electricity, an information channel is also organized simultaneously. The use of contactless power transmission for charging AUV batteries in automatic mode in the case of its underwater basing has almost no alternative [5].

The components of the contactless charging system are a DC-AC converter (inverter), a high-frequency transformer with separated primary $\mathrm{T} 1$ and secondary $\mathrm{T} 2$ parts, a battery charger (BC) and a rechargeable battery (RB). The placement of the functional elements of the AUV power supply system and the connection between them when using the bottom dock-station is shown in figure 1 .

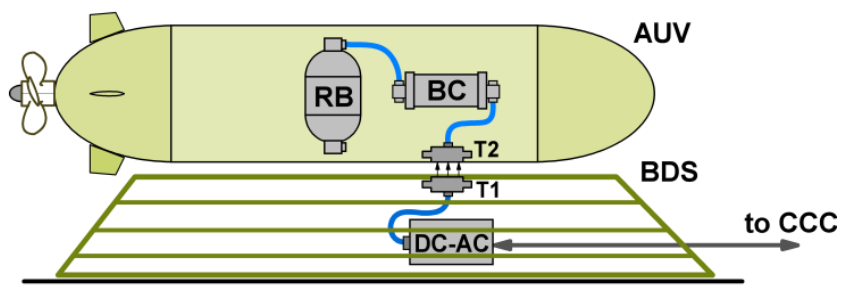

Figure 1. Functional diagram of a contactless transmission system electric power at AUV from dock-station

The primary $\mathrm{T} 1$ and secondary $\mathrm{T} 2$ parts are separate sealed shells in which the transformer windings are placed. In the energy transfer mode, the axes of the windings must coincide, and their ends must be at a minimum distance from each other. Each of the shells is made of an insulating material, the thickness of which reaches several millimetres. Due to the presence of this non-magnetic constructive gap between the end surfaces of the windings, such a transformer has a low value of the magnetic coupling coefficient between the windings [6].

This causes an increased value of the magnetizing component of the transformer input current. In this case, the output current of the inverter increases several times compared to the minimum required value determined by the load of the transformer on its secondary side. Generally, this causes consequences such as the need to select transistors and diodes of the inverter with high rated currents; increasing power losses in these semiconductor devices, increasing their weight and cost; complicating the problem of heat dissipation caused by additional power losses.

The negative nature of these consequences is enhanced in the energy supply system of the underwater vehicle, since the electronic units must be placed in sealed containers with a tight layout and with limited heat removal capabilities from power devices.

These circumstances determine the relevance of the search for solutions that will reduce the current load on the inverter power switches while maintaining the set value of the transmitted active power. In the end, this should lead to an increase in the device reliability and increase the efficiency of the contactless battery charge system of the underwater vehicle as a whole.

In general, there are three main ways to reduce the inverter load: increasing the transformer coupling coefficient [7-9], compensating the transformer reactive input current with resonant circuits, and providing softswitching of inverter power transistors [10].

The first direction is mainly related to increasing the contact areas of the transformer primary and secondary parts. However, this path is limited by the requirements for placing the receiving part on the AUV and in some cases is not possible.

The second direction is more flexible. It allows not only to unload the inverter, but also to coordinate the contactless power transmission transformer with the set voltage level of the source, and with the set electrical parameters of the load. This simplifies the transformer design. In addition, using resonant circuits, it is possible to facilitate the receiving part of the charge system [11].

Frivaldsky et al. [12] gave an overview of the main performance characteristics of the most common resonant circuit variants. Thus, it is concluded that all circuits with a serial capacitor in the transmitting part are able to increase the transmitted power to the load. Parallel capacitor circuits allow working with maximum efficiency when the transmitted power is maximum. The study covers the system behavior only in the selected frequency range and for a given load resistance. However, the resonant circuit efficiency should be determined for the entire range of load variation. In $[11,13,14]$, various versions of resonant circuits were proposed that provide both compensation of the reactive current and switching at zero voltage (ZVS). However, these circuits contain additional components that increase the size, weight and cost of the contactless power transmission system. In these articles, resonant circuits are mainly intended to provide ZVS mode. At the same time, certain losses are caused by switching off at a non-zero current. It should be considered that ensuring shutdown at zero current is also important.

This article will analyze the inverter operation at idle on the secondary side of the transformer, as well as in load mode. The purpose of the analysis is to determine the relationship between the parameters of the resonant circuit elements, which allow reducing heat losses in the inverter switches.

\section{Analysis of the inverter operation at idle on the secondary side of the transformer}

The solution to the problem of increasing the efficiency of the contactless charge system can be obtained by using resonant circuits on both the primary side of the transformer and the secondary side, in combination with 
the soft switching mode. The implementation of such RC1 and RC2 circuits, shown in figure 2, may be different, but the final result should be determined by the sum of the introduced effects without mutual influence. This will help to adequately reproduce the results of the calculation of resonant elements in their practical implementation and simplify the overall configuration of the system.

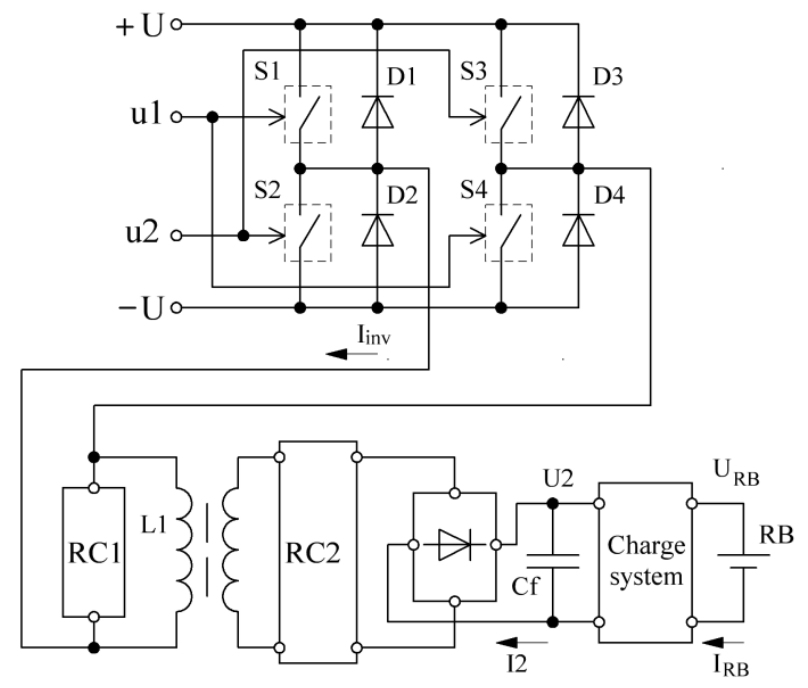

Figure 2. Block diagram of the contactless charge system

Generally, the total power loss on the inverter switches is calculated from the dynamic power loss on and off, as well as the static loss in the open state of the switch. Losses from leakage currents in the closed state are small and can be ignored. In [15], the dynamic losses are minimized when switching on. This is achieved by creating conditions for switching on at zero voltage on the transistor - the ZVS mode (zero voltage switching mode). This mode is called "soft switching". At the same time, calculations and experiments show that both dynamic losses during shutdown and power losses on switches in the open state also make a significant contribution to the total amount of losses. Thus, it is possible to set the actual task of providing conditions for "soft switching" when switching off, which is achieved, obviously, by switching off at zero current - the ZCS mode (zero current switching mode). At the same time, it is important to reduce the loss of conductivity in the open state interval of switches. Note that for all types of corrective actions on the system, the value of the transmitted active power must remain unchanged.

The solution to the problem of current unloading of switches in static mode can be found, obviously, in the form of such a structure RC1, which compensates the magnetizing component of the input current of the transformer. Since this component has an inductive nature, the compensation should be performed by some element that has a capacitive nature of the load. The use of a capacitor in its pure form is limited here, since a rectangular voltage is formed at the output of the inverter.
One of the well-known methods of current unloading of power switches in static mode is the use LC low-pass filter at the output of the inverter. In this case, the inverter load (the primary winding of the transformer) is connected in parallel to the filter capacitor [16-19]. This solution allows unload the inverter switches. However, the external characteristic of the transformer is distorted due to voltage drop at the reactor, and, as a result, the transmitted power is reduced.

The best results are obtained using a serial resonant LC-circuit connected to the inverter output parallel to the transformer primary winding [17]. This circuit also compensates the inductive component of the inverter output current due to its capacitive nature, but practically does not affect the shape and values of the transformer winding currents. This reduces the current value of the inverter output current while maintaining the level of transmitted active power. The applied resonant circuit has its own resonant frequency $\mathrm{f}_{1 \mathrm{U}}$, corresponding to the voltage resonance [18]

$$
f_{1 U}=\frac{1}{2 \pi \sqrt{L_{R} \cdot C_{R 1}}},
$$

where $L_{R}$ and $C_{R 1}$ - inductance and capacitance of the resonant circuit on the primary side of the transformer, respectively.

At the same time, the inverter operating frequency $f$ must correspond to the current resonance frequency $f_{1 I}$ in the inverter load, which consists of a parallel resonant serial LC-circuit and the transformer primary winding $\mathrm{L}_{1}$. In this condition, the minimum current load on the inverter transistor switches is provided. For the first harmonic of the $\mathrm{AC}$ voltage at the output of the inverter, the ratio is true

$$
f=f_{1 I}=\frac{1}{2 \pi \sqrt{\left(L_{R}+L_{1}\right) C_{R 1}}},
$$

where $\mathrm{L}_{1}$ - transformer primary inductance.

If we limit the analysis conditions to the first harmonic of the inverter output oscillations, then the reactor inductance $L_{R}$ and the capacitance $C_{R 1}$ of the series resonant circuit capacitor $\mathrm{RC} 1$ can be determined by the formulas [18]

$$
\begin{gathered}
L_{R}=\frac{L_{1}}{m^{2}-1}, \\
C_{R 1}=\frac{m^{2}-1}{(2 \pi \cdot m \cdot f)^{2} L_{1}},
\end{gathered}
$$

where parameter $\mathrm{m}=\mathrm{f}_{1 \mathrm{U}} / \mathrm{f}_{1 \mathrm{I}}$ - ratio of the natural frequency of the resonant LC-circuit (voltage resonance frequency) to the inverter switching frequency (current resonance frequency of the inverter load circuit). The choice of the m-parameter value is determined by the compromise between the desired decrease of the inverter switches current and the acceptable reactive power values of the capacitance and inductance of the resonance circuit, which determines the mass and dimensions of these elements. 
To obtain the current resonance in the load of the inverter for the first harmonic of its output voltage, the value of the relative frequency must be equal to unity. Since a rectangular signal is generated at the inverter output, in order to obtain the minimum effective value of the inverter output current, the coefficient $n_{1 I}$ should be slightly more than one. This value of the relative frequency $n_{1 I}$ was found by mathematical modelling in the MATLAB program using the models given in $[18,20]$.

To analyse the obtained inverter current time diagrams $I_{\text {inv }}(t)$, we will take the numbering of the inverter switches and opposite diodes, as shown in Figure 2. The simulation results for the transformer idle mode, illustrating the effect of including a resonant LC-circuit RC1, are shown in Figure 3.

The inverter control signals $\mathrm{u}_{1}$ and $\mathrm{u}_{2}$ arrive at the diagonally located switches. The signals have a dead time interval when switching. This prevents emergency currents through the switches of one rack S1, S2 or S3, $\mathrm{S} 4$. The graphs in Figure 3a correspond to the state when there is no resonant LC-circuit and only the transformer primary winding with its own inductance $L_{1}$ is connected to the inverter output. In this case, the current in the load changes linearly and flows in turn through the diagonal switches or through the diodes. Figure $3 \mathrm{a}$ shows the conduction intervals of the switches (double line) and diodes (dashed lines) for this case during one switching period. The control signal $\mathrm{u}_{1}$ affects the inputs of the switches $S 1, S 4$ in the time interval $t_{1}-t_{2}$, then follows the "dead time" in the interval $t_{2}-t_{3}$. Next, control $u_{2}$ is supplied to the switches S2, S3 of the second inverter diagonal bridge in the time interval $t_{3}-t_{4}$. The intervals of the switches and diodes conductivity vary, while the switches of each diagonal conduct current when exposed to the corresponding control signal $\mathrm{u}_{1}$ or $\mathrm{u}_{2}$ after the interval of the diodes conductivity.

If the inverter current is taken as a unit value at the moments when the switches are closed for operation without a resonant circuit, the inverter current effective value (triangular current shape) will be $1 / \sqrt{ } 3$. This is an increased value with respect to the mode with resonance. In addition, as follows from Figure $3 \mathrm{a}$ ), at the time of closing the switches (moment $\mathrm{t}_{2}$ for $\mathrm{S} 1, \mathrm{~S} 4$ and moment $\mathrm{t} 4$ for S2, S3), the current through the switches has a maximum value. This leads to significant power losses on the switches in the off mode, problems of heat dissipation and increased heating of the inverter power elements.

When series resonant LC-circuit (RC1) is connected in parallel with the transformer primary winding, the shape of the inverter output current and the conduction intervals of the switches and diodes change. For the first harmonic of the inverter output voltage, the condition $n_{1 I}=f / f_{1 I}=1$ corresponds to the currents resonance in its load. In this case, the effective value of the inverter output current has a minimum value determined by the active losses in the current flow circuits. For the actual inverter output signal in the form of a meander, the minimum effective value of the inverter current is reached at $n_{1 I}=f / f_{1 I}>1$. The shape of the inverter output current and the conduction intervals of the switches and diodes will have the form, as shown in Figure $3 b$ ).

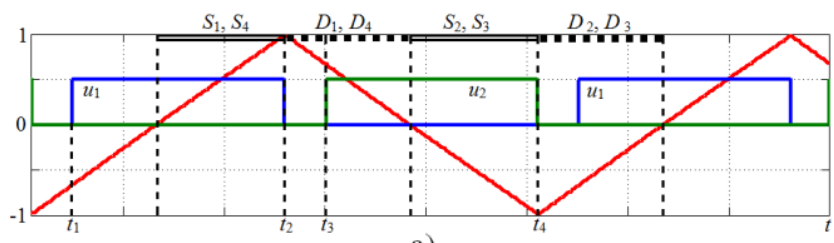

a)

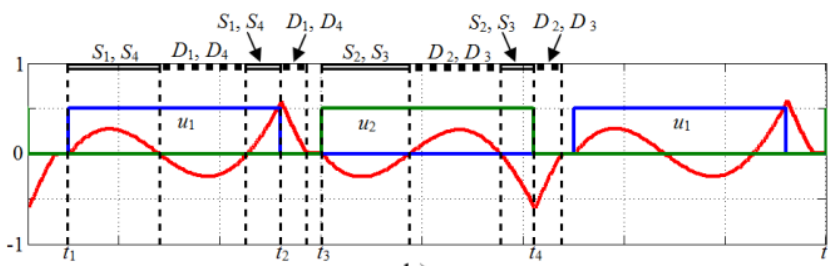

b)

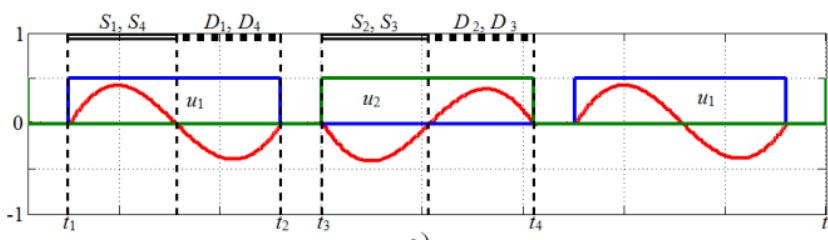

c)

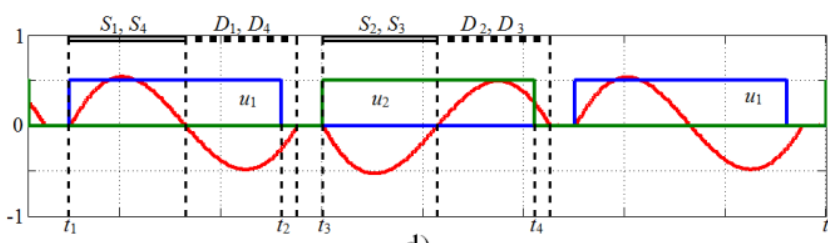

d)

Figure 3. Inverter output current diagrams when changing the resonant circuit parameters:

a - resonant circuit none; b - resonant circuit parameters correspond to the relative frequency $\mathrm{n}_{1 /}=1 ; \mathrm{c}, \mathrm{d}$ - resonant circuit parameters correspond to zero switching losses for $n_{1 /}>1$.

The simulation results show that in this way it is possible to reduce the effective value of the inverter current by about 2.5 times [18, 19]. However, a corresponding reduction in power loss does not occur here. The reason for this is the shape of the inverter current, in which, like the original version, at the time $t_{2}$ and $t_{4}$ of switching off the transistors, the current is significant. This value is less than the amplitude of the sawtooth current in Figure 3a), and is approximately 0.6. But this turns out to be enough so that the total power loss in the inverter remains at a high level.

A significant reduction in the inverter power loss can be obtained by "soft switching", which will be performed at a certain value of the relative frequency $n_{1 I}>1$ (Figure $3 c)$ ). In this mode, the inverter transistors will turn off at zero current and will be accompanied by minimal dynamic losses. An increase in the relative frequency of the current resonance will deform the curve of the inverter output current, as shown in Figure 3d). In this case, the soft switching mode will remain with an increase in the 
diodes conductivity interval D1, D4 until the switches S2, S3 are opened, that is, in the "dead time" interval. A further increase the value $\mathrm{n}_{1 \mathrm{I}}$ will lead to an increase in open conductivity losses from the increased capacitive component of the inverter load current due to RC1. Here, the numbering of the diodes and switches is given for one half-cycle of the inverter output signal. For the second half-cycle, in accordance with Figure 2, diodes D2, D3 and switches S1, S4 will participate in the discussion.

Thus, there is a compromise problem of determining the relative frequency value $\mathrm{n}_{1 \mathrm{I}}$, which minimizes losses to the inverter both in the transformer idle mode and in the full range of its loads, including the consequences of inaccurate joining of the transmitting $\mathrm{T} 1$ and receiving $\mathrm{T} 2$ transformer parts when the AUV approaches the base. At the same time, both switching losses and conductivity losses should be minimized.

To solve this problem, mathematical simulation of the inverter was performed. The dependences of the current $\mathrm{I}_{\mathrm{OFF}}{ }^{*}$ through the inverter transistors at the time of the control signal for shutdown, the effective values of the inverter current $\mathrm{I}_{\mathrm{INV}}{ }^{*}$ and the power losses $\mathrm{P}_{\mathrm{INV}}{ }^{*}$ in the inverter depending on the relative frequency $n_{1 I}=f / f_{1 I}$ were obtained. The results are shown in Figure 4, where the variables are shown in relative units. The values of these variables are taken as unity in the absence of the $\mathrm{RC} 1$ resonant circuit.

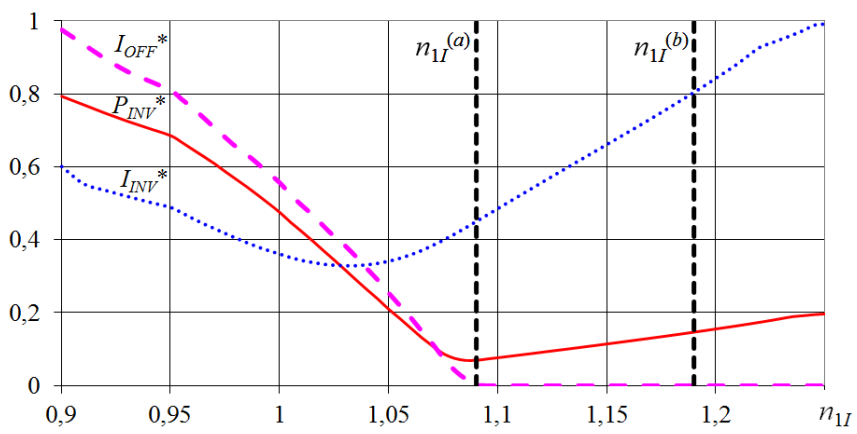

Figure 4. Inverter frequency characteristics in the idle mode:

$\mathrm{I}_{\mathrm{OFF}}{ }^{*}$ - current through the switches at the time of shutdown; $P_{I_{N V}}{ }^{*}$ - inverter power loss; $I_{I N V}{ }^{*}$ - inverter output current effective value.

From these graphs it follows that the current value $\mathrm{I}_{\mathrm{OFF}}{ }^{*}$ and, accordingly, the loss during shutdown reach zero at a relative frequency $\mathrm{n}_{1 \mathrm{I}}{ }^{\text {(a) }}=1.09$. The area on the graph, located to the right of the frequency $\mathrm{n}_{1 \mathrm{I}}{ }^{(\mathrm{a})}$, corresponds to the ZCS area (switching at zero current). Here, zero shutdown losses are stored. To the left of the frequency $\mathrm{n}_{1 \mathrm{I}}{ }^{(\mathrm{b})}=1.19$ is the ZVS region (switching at zero voltage), which corresponds to the inductive load mode of the inverter and is characterized by zero switching losses.

Thus, the range of relative frequency values from $\mathrm{n}_{1 \mathrm{II}}{ }^{\text {(a) }}$ $\geq 1.09$ to $\mathrm{n}_{1 \mathrm{I}}^{(\text {b) }} \leq 1.19$, obtained by mathematical modelling, corresponds to the end of the diodes conductivity interval D1, D4 (or diodes D2, D3) in the range of the dead time interval (Figure $3 \mathrm{~d}$ )). This range provides low power losses, both on switching on and off the inverter transistors. It is advisable to take the value of the relative frequency from this range closer to its left border, for example, $\mathrm{n}_{1 \mathrm{I}}=1.1$. So the total losses on the transistors will be minimal. Keep in mind that when choosing the value of the relative frequency, the switching losses remain zero, and the conductivity losses increase slightly due to an increase in the inverter output current.

The graphs in Figure 4 are defined for some $m=f_{1 U} /$ $\mathrm{f}_{1 \mathrm{I}}=$ const and correspond to certain values of the reactor inductance $L_{R}$ and capacitance $C_{R 1}$ of the resonance circuit $\mathrm{RC} 1$. At the same time, it is of practical interest to determine the value of the parameter $\mathrm{m}$ at which the powers of these elements are minimal while maintaining minimal losses in the inverter. For this, it is possible to use the dependences of the relative powers of the reactor $\mathrm{S}_{\mathrm{L}}{ }^{*}$ and capacitance $\mathrm{S}_{\mathrm{C}}{ }^{*}$ of the resonance circuit $\mathrm{RC} 1$ in the function of parameter $\mathrm{m}$, provided that it minimizes losses in the inverter. These values are determined in relation to the inverter power without the $\mathrm{RC} 1$ resonant circuit.

Figure 5 shows the graphs of the relative powers $\mathrm{S}_{\mathrm{L}}{ }^{*}$, $\mathrm{S}_{\mathrm{C}}{ }^{*}$ as a function of the parameter $\mathrm{m}$, as well as the dependences of the inverter relative power $S_{\text {INV }}{ }^{*}$ and the relative losses $\mathrm{P}_{\mathrm{INV}}{ }^{*}$ in the inverter when the resonant circuit RC1 is connected.

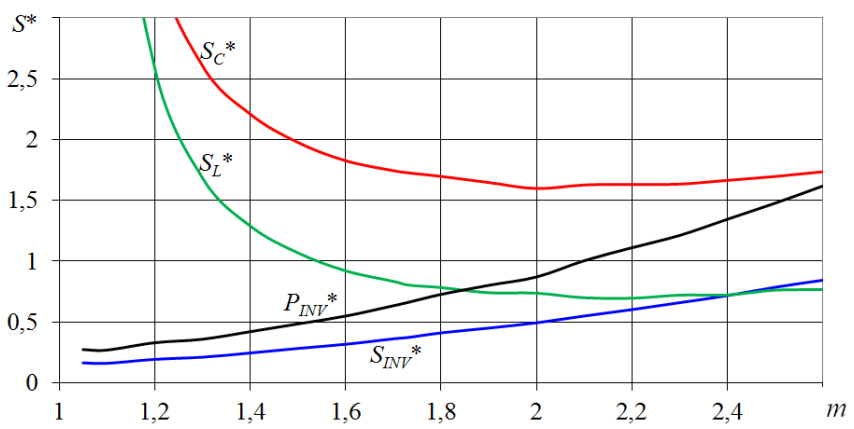

Figure 5. Relative power dependences of the inverter and the elements of the resonant LC-circuit on the parameter $m$ at idle: $S_{I_{N V}}{ }^{*}-$ inverter output power; $P_{I N V}{ }^{*}$ - power losses in inverter; $S_{C}{ }^{*}, S_{L}{ }^{*}$ capacitor and reactor capacities, respectively.

From figure 5 it follows that the power and, accordingly, the dimensions of the inductance and capacitance reach the minimum values at $2<\mathrm{m}<2.2$. Taking the parameter $\mathrm{m}$ in this range (for example, $\mathrm{m}=$ 2 ), it is possible to determine the required values of $L_{R}$ and $C_{R 1}$ of the resonance circuit.

Note that the above considerations apply to the transformer idling and the connection of the load does not change the mode of operation of its primary side. 


\section{Characteristics of the contactless power transmission system in load mode on the secondary side}

When a load transformer is connected to the secondary winding, the parameters of the electric energy transferred to it can be determined in the form of external characteristics of the system $\mathrm{U}_{2}\left(\mathrm{I}_{2}\right)$ (voltage $\mathrm{U}_{2}$ and current $I_{2}$ are shown in Figure 2). The load in this case is a combination of a rectifier, a filter $\mathrm{C}_{\mathrm{f}}$, a pulse converter with an automatic control system (charge system) and battery. A full charge of the battery will be possible if a input voltage of the charge system is greater than the voltage of a fully charged battery. The wide possibilities of deformation of the system initial external characteristic in the desired direction are presented when an RC2 resonant circuit is introduced on the transformer secondary side. Of the possible circuit implementations, the practical interest is the implementation of $\mathrm{RC} 2$ in the form of a capacitor $\mathrm{C}_{\mathrm{R} 2}$, connected in series with the transformer secondary winding. Under certain conditions, it can be effective to turn on the resonant capacitor $C_{R 2}$ parallel to the transformer secondary winding.

When connected in series with the transformer primary winding of the capacitor $\mathrm{C}_{\mathrm{R} 2}$, the external characteristic of the electric energy transmission system $\mathrm{U}_{2}\left(\mathrm{I}_{2}\right)$ can take the form of one of the characteristics shown in figure 6 , where $\mathrm{U}_{2}{ }^{*}$ is the voltage at the charge system input, $\mathrm{I}_{2}{ }^{*}$ is the charge system input current in relative units, while the no-load voltage and short-circuit current for this graph are taken as single base values. A family of characteristics is obtained when the value of the resonant capacitor $C_{R 2}$ changes [21, 22].

Connecting a resonant capacitor to the secondary circuit deforms the external characteristics of the system, as shown in Figure 6 . The capacitance value $C_{R 2}=C_{1}$ corresponds to the voltage resonance. A decrease in capacitance with respect to this value increases the inclination of the external characteristics.

Practical applications have characteristics in the capacitance range of a resonant capacitor $\mathrm{C}_{2}>\mathrm{C}_{\mathrm{R} 2}>\mathrm{C}_{3}$. We can assume that the external characteristic corresponding to the capacitor $\mathrm{C}_{3}$ is best suited for the energy transfer process. The form of this characteristic has a rational combination of a section with high offset (current limiting) and a section with low offset (voltage limiting). This corresponds closely enough to the charging algorithm of lithium-ion batteries. The effect of introducing a series resonant capacitor is to increase the system efficiency as a whole while minimizing the mass and dimensions of the transformer.

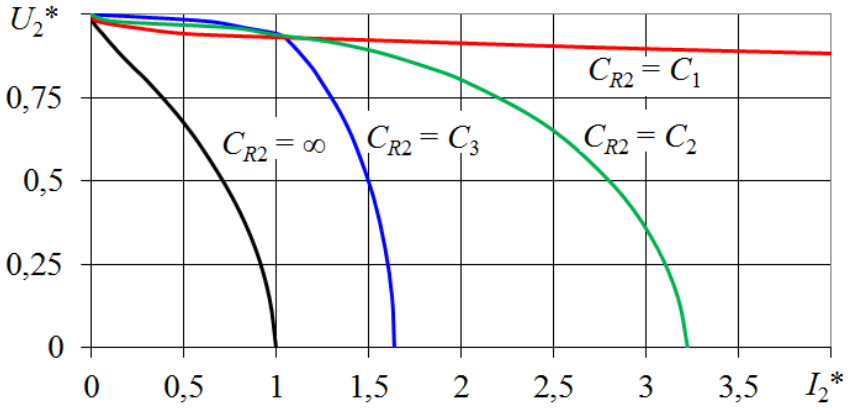

Figure 6. External characteristics of the power transmission system for different values of the resonant capacitor $\mathrm{C}_{\mathrm{P}}$ in the load circuit:

$$
\mathrm{C}_{\mathrm{R} 2}=\mathrm{C}_{1}>\mathrm{C}_{2}>\mathrm{C}_{3}
$$

The choice of a specific capacitance value $C_{R 2}$ can be justified when analysing the frequency characteristics of the secondary circuit, shown in Figure 7. It shows the dependences of the transmitted active power $\mathrm{P}_{2}{ }^{*}$ and the overall power $\mathrm{S}_{\mathrm{C}}{ }^{*}$ of this capacitor in relative units on the relative resonant frequency of the current $n_{2 I}=f / f_{2 I}$. In this case, the variables $\mathrm{P}_{2}{ }^{*}$ and $\mathrm{S}_{\mathrm{C}}{ }^{*}$ are determined in relation to the transmitted power without a resonant capacitor.

The values of the variables $\mathrm{P}_{2}{ }^{*}$ and $\mathrm{S}_{\mathrm{C}}{ }^{*}$ increase to infinity at the voltage resonance frequency

$$
f_{2 U}=\frac{1}{2 \pi \sqrt{\left(1-k^{2}\right) L_{2} C_{R 2}}},
$$

where $\mathrm{C}_{\mathrm{R} 2}, \mathrm{~L}_{2}$ - the capacitance of the resonant capacitor and the secondary winding inductance, respectively; $\mathrm{k}$ - is the magnetic coupling coefficient of the transformer [6].

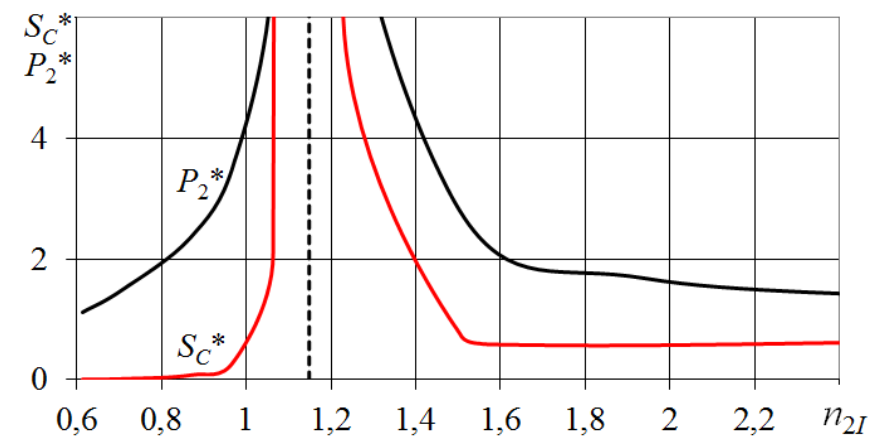

Figure 7. System secondary circuit frequency characteristic with series connection of the resonant capacitor

The current resonance frequency for the secondary circuit of the system is determined by

$$
f_{2 I}=\frac{1}{2 \pi \sqrt{L_{2} C_{R 2}}} .
$$

Based on (5) and (6), the current resonance frequency $f_{2 I}$ for the secondary circuit of the system will always be less than the voltage resonance frequency $\mathrm{f}_{2 \mathrm{U}}$.

According to the graphs shown in Figure 7, it is possible to establish a relationship between the relative 
frequency of the current resonance $\mathrm{n}_{21}=\mathrm{f} / \mathrm{f}_{2 \mathrm{I}}$, the corresponding active power $\mathrm{P}_{2}{ }^{*}$ transmitted by the system and the overall power $\mathrm{S}_{\mathrm{C}}{ }^{*}$ of the resonant capacitor. The values of these variables are decisive in solving the compromise problem of choosing the resonant frequency $\mathrm{n}_{21}$.

So, for example, if a decision is made to increase the transmitted power three times, then, in accordance with Figure 7, this will require the use of a series resonant capacitor with a relative overall power of approximately $\mathrm{S}_{\mathrm{C}}{ }^{*} \approx 0.1$. The selected mode corresponds to the relative current resonant frequency $\mathrm{n}_{2 \mathrm{I}} \approx 0.93$. This value, according to formula (6), allows determining the capacitance of the series resonant capacitor $\mathrm{C}_{\mathrm{R} 2}$.

Obviously, other decisions can be here, depending on the task and the priorities. For example, if it required a large increase in the transmitted power, then using the graphs in figure 7 , we can find the values of the relative frequency of the current resonance and the overall power of the capacitor. Overall power in this case will also matter more.

It is important to note that the proposed approach to increasing the efficiency of the contactless charge system has such a benefit as the possibility of independent influence on the operating modes of the transformer primary and secondary sides. Indeed, using the RC1 resonant circuit from the capacitance and inductance connected in series and providing soft switching of the inverter transistors on the primary side, we obtain a significant tenfold reduction in the power loss on power switches. Adding to this solution the RC2 resonant circuit in the form of a capacitor connected in series with the transformer secondary winding, we get the possibility of independent formation of the external characteristics of the transmission system. A certain (optimal) capacitance value of such a capacitor allows reducing the transformer weight and dimensions, which is relevant for AUV.

In some cases, a $\mathrm{C}_{\mathrm{R} 2}$ capacitor connected in parallel with the transformer secondary winding can be used as a resonant circuit of RC2. In this case, the voltage and current resonance on the secondary side will also be determined by expressions (5) and (6), respectively, and the graphs of the external characteristics will take the form, as shown in Figure 8.

The initial external characteristic of the system at $\mathrm{C}_{\mathrm{R} 2}=$ 0 has an ordinate equal to one at idle $\left(\mathrm{I}_{2}{ }^{*}=0\right)$. An increase in the capacitance of the $\mathrm{C}_{\mathrm{R} 2}$ capacitor leads to an increase in the output voltage of the system, which is illustrated by the characteristics in Figure 8. Voltage resonance occurs, and the ordinate of the external characteristic rushes to infinity, when $\mathrm{C}_{\mathrm{R} 2}=\mathrm{C}_{3}$.

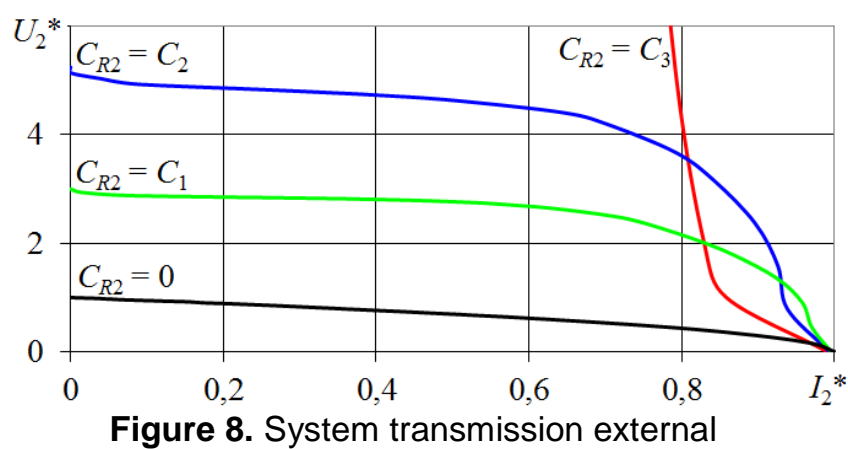

characteristics at parallel connection of the resonant capacitor on the transformer secondary side:

$$
\mathrm{C}_{1}<\mathrm{C}_{2}<\mathrm{C}_{3}
$$

If it is necessary to have an increased voltage on the secondary side of the transformer, the use of a resonant circuit RC2 in the form of a parallel capacitor $\mathrm{C}_{\mathrm{R} 2}$ may be appropriate. In general, this goal can be achieved by increasing the number of transformers in the system with the inclusion of their secondary windings in series. This solution may be problematic due to the equipment location in the AUV compartment. The solution may be the use of the specified resonant circuit, which will allow providing the required mode with the performance of dimensions restrictions.

At the same time, the decision must be considered and justified. If the number of transformers is limited, it is necessary to simultaneously solve the problem of placing a resonant capacitor.

Selecting a specific capacitance value for a parallel capacitor can be performed similar to the same task for a serial resonant capacitor. To do this, consider the frequency characteristics of the secondary circuit in figure 9. The figure shows the dependencies of the transmitted active power $\mathrm{P}_{2}{ }^{*}$ and the overall power $\mathrm{S}_{\mathrm{C}}{ }^{*}$ of this capacitor in relative units on the relative resonant frequency of the current $n_{2 I}=f / f_{2 I}$. In this case, the variables $\mathrm{P}_{2}{ }^{*}$ and $\mathrm{S}_{\mathrm{C}}{ }^{*}$ are determined in relation to the transmitted power without a resonant capacitor.

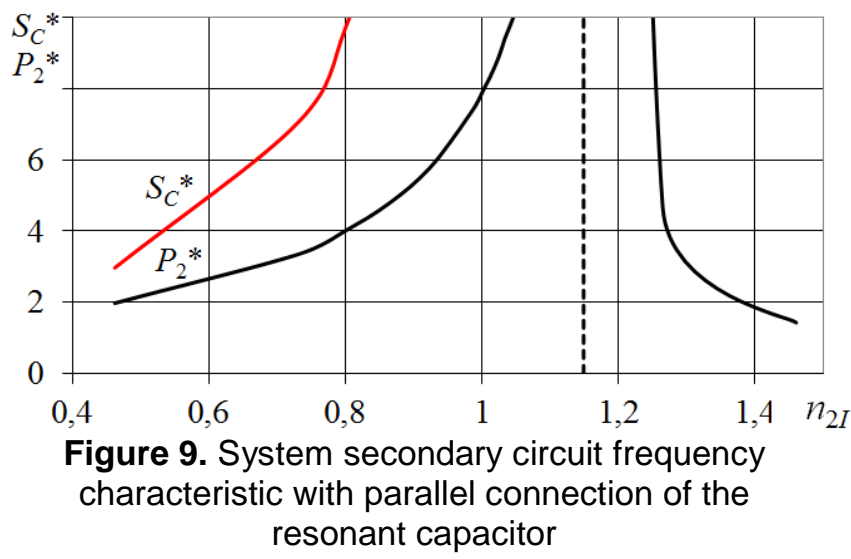

Here, it should also specify the desired increase in the transmitted active power $\mathrm{P}_{2}{ }^{*}$ in relation to the same parameter of the system without resonance. This determines the relative current resonant frequency and, 
further, the overall power of the resonant capacitor. The found value $n_{2 I}=f / f_{2 I}$ using the expression (6) allows determining the capacity of the resonant capacitor.

Thus, the solutions considered, consisting in the use of resonant elements on both the primary and secondary sides of the transformer, are accompanied by the summation of positive properties. Indeed, the introduction of a resonant LC-circuit parallel to the transformer primary winding allows reducing losses in the inverter, reducing the power switches temperature and increasing the device reliability without affecting the system external characteristics. The use of a serial (or parallel) resonant capacitor on the transformer secondary side leads to the independent formation of the required external characteristic that determines the transmitted power. However, restrictions related to the permissible loads on the resonant capacitor $C_{R}$ will already apply [23].

\section{Conclusion}

Research has shown that the use of the resonant LCcircuit on the transformer primary side and a series resonant capacitor on its secondary side in combination with the soft-switching mode reduced the losses. In the transformer idle mode, the losses in the inverter are reduced from $170 \mathrm{~W}$ to $25 \mathrm{~W}$. In the transformer shortcircuit mode, the losses in the inverter are reduced from $170 \mathrm{~W}$ to $25 \mathrm{~W}$. Using these solutions promotes to increase the transmitted power from $212 \mathrm{~W}$ to $570 \mathrm{~W}$. These results indicate a significant increase in the system quality characteristics and minimize the AUV battery charge time. At the same time, the proposed solution provides the specified performance with a limited range of inverter load changes. Outside of this load range, softswitching mode is terminated and losses in the inverter increase. Further system improvement involves the use of duty cycle and frequency automatic control system of the inverter, which is the subject of further research by the authors.

\section{References}

[1] X. Wang, J. Shang, Z. Luo, L. Tang, X. Zhang, J. Li. Reviews of power systems and environmental energy conversion for unmanned underwater vehicles. Renewable and Sustainable Energy Reviews. 2012, vol. 16, is. 4, pp. 1958-1970.

[2] Stanimir S. Valtchev, Elena N. Baikova, Luis R. Jorge Electromagnetic Field as the Wireless Transporter of Energy. Facta Universitatis, Ser: Elec. Energ. December 2012, vol. 25 , no. 3 , pp. $171-181$.

[3] S.K. Singh, T.S. Hasarmani, R.M. Holmukhe. Wireless Transmission of Electrical Power Overview of Recent Research and Development. International Journal of Computer and Electrical Engineering. 2012, pp. 207-211.

[4] Patent. 2502170, Russian Federation. Device for noncontact transfer of electric energy to underwater object (versions). Gerasimov V.A., Kopylov V.V., Kuvshinov
G.E., Naumov L.A., Filozhenko A.Yu., Chepurin P.I. Date of publication: 20.12.2013, bull. 35 .

[5] Gerasimov V.A., Filozhenko A.Yu., Chepurin P.I. Structure of the system noncontact energy issue of the autonomous undersea device. Izvestiya SFedU, Engineering sciences. 2013, no. 3 (140), pp. 47-55.

[6] Gerasimov V.A., Kopilov V.V, Kuvshinov G.E., Naumov L.A., Sebto Y.G., Filozhenko A.Yu, Chepurin P.I. Mathematical modeling for contactless electric power transfer to underwater vehicle. Underwater investigations and robotics. Vladivostok, 2012, no. 2 (14), pp. 28 -33.

[7] Haibing, W., Baowei, S., Kehan, Z., \& Zhengchao, Y. (2018). A novel electromagnetic actuator in an inductive power transmission system for autonomous underwater vehicle. Advances in Mechanical Engineering, 10(9), 168781401879742. DOI:10.1177/1687814018797421.

[8] Kim, S.; Covic, G.A.; Boys, J.T. Comparison of Tripolar and Circular Pads for IPT Charging Systems. IEEE Trans. Power Electron. 2018, 33, 6093-6103. DOI: 10.1109/TPEL.2017.2740944.

[9] Kan, T., Mai, R., Mercier, P. P., \& Mi, C. C. (2018). Design and Analysis of a Three-Phase Wireless Charging System for Lightweight Autonomous Underwater Vehicles. IEEE Transactions on Power Electronics, 33(8), 6622-6632. DOI:10.1109/tpel.2017.2757015.

[10] Liu, X., Wang, T., Jin, N., Habib, S., Ali, M., Yang, X., \& Tang, H. (2018). Analysis and Elimination of Dead-Time Effect in Wireless Power Transfer System. Energies, 11(6), 1577. DOI:10.3390/en11061577.

[11] Yan Z.; Zhang Yi.; Song B.; Zhang K.; Kan T.; Mi C. An LCC-P Compensated Wireless Power Transfer System with a Constant Current Output and Reduced Receiver Size. Energies 2019, 12(1), 172. DOI: 10.3390/en12010172.

[12] Frivaldsky, M., Spanik, P., Drgona, P., Jaros, V., \& Piri, M. (2016). Analysis of Wireless Power System Efficiency in Dependency on Configuration of Resonant Tank. Wireless Power Transfer - Fundamentals and Technologies. DOI: 10.5772/62998.

[13] Wang, Y., Yao, Y., Liu, X., \& Xu, D. (2017). S/CLC Compensation Topology Analysis and Circular Coil Design for Wireless Power Transfer. IEEE Transactions on Transportation Electrification, 3(2), 496-507. DOI: 10.1109/tte.2017.2651067.

[14] Li, S.Q.; Li,W.H.; Deng, J.J.; Nguyen, T.D.; Mi, C.C. A Double-Sided LCC Compensation Network and Its Tuning Method for Wireless Power Transfer. IEEE Trans. Veh. Technol. 2015, 64, 2261-2273. DOI: 10.1109/TVT.2014.2347006.

[15] Ching-Ming Lai, Ming-Ji Yang, Shih-Kun Liang. A Zero Input Current Ripple ZVS/ZCS Boost Converter with Boumdary-Mode Control. Energies. 2014, pp. 6765-6782.

[16] Gerasimov V.A., Kraskovskiy M.V., Filozhenko A.Yu. Unloading transistors of the inverter in the system contactless transmission on autonomous underwater vehicle. Izvestiya SFedU, Engineering sciences. 2016, no. 4 (177), pp. $147-158$.

[17] Patent. 2558681, Russian Federation. Independent voltage inverter to supply load through transformer with low coupling coefficient between its windings. Gerasimov V.A., Kuvshinov G.E., Naumov L.A., Sebto Yu. G., Filozhenko A.Yu., Chepurin P.I. Date of publication: 10.08.2015, bull. 22

[18] Gerasimov V.A., Kraskovskiy M.V., Kuvshinov G.E., Filozhenko A.Yu. Using a resonance for current discharging of transistors switch in the inverter. Dvoynyye 
tekhnologii [Dual Technologies]. 2016, no. 4 (77), pp. 55 - 60. (In Russian)

[19] Gerasimov V.A., Kraskovskiy M.V., Kuvshinov G.E., Filozhenko A.Yu. The Use of Resonance for Current Downloading of the Transistor Keys of the Inverter. International Journal of Control Theory and Applications. 2016, vol. 9, is. 13, pp. 305-311.

[20] Gerasimov V.A., Kraskovskiy M.V., Kuvshinov G.E., Filozhenko A.Yu. Improving the efficiency contactless transmission electricity on autonomous underwater vehicle. Underwater investigations and robotics. Vladivostok. 2016, no. 1 (24), pp. $24-30$.

[21] Patent. 2530877, Russian Federation. Charging device for accumulator battery. Gerasimov V.A., Filozhenko A.Yu., Chepurin P.I. Date of publication: 20.10.2014, bull. 29.

[22] Patent. 2602078, Russian Federation. Device for underwater object storage battery charging. Gerasimov V.A., Filozhenko A.Yu. Date of publication: 10.11.2016, bull. 31.

[23] Metallized Polypropylene Film Capacitors. Series/Type: B32656S ... B32658S. TDK. October 2014. Available at: http://www.ic-

contract.ru/images/pdf/TDK/MKP_B32656S.pdf (accessed 20 March 2020). 